Research/Technical Note

\title{
Revascularization of Temporomandibular Joint After Surgical Intervention Using CT Volume Rendering Technique (Clinical Study)
}

\author{
Heba Abdul Wahed Sleem ${ }^{1,}$, Mona Gamalludin Alkaphoury ${ }^{2}$ \\ ${ }^{1}$ Department of Oral and Maxillofacial Surgery, Faculty of Dentistry Ain Shams University, Cairo, Egypt \\ ${ }^{2}$ Department of Diagnostic Radiology, Faculty of Medicine, Ain Shams University, Cairo, Egypt
}

Email address:

drsleemh@gmail.com(H. A. W. Sleem)

${ }^{*}$ Corresponding author

\section{To cite this article:}

Heba Abdul Wahed Sleem, Mona Gamalludin Alkaphoury. Revascularization of Temporomandibular Joint After Surgical Intervention Using CT Volume Rendering Technique (Clinical Study). International Journal of Clinical Oral and Maxillofacial Surgery.

Vol. 4, No. 1, 2018, pp. 25-29. doi: 10.11648/j.ijcoms.20180401.15

Received: July 1, 2018; Accepted: July 17, 2018; Published: August 27, 2018

\begin{abstract}
The present study aimed to trace the arterial blood supply of Tempromandibular joint after open joint procedures using CT volume rendering technique. To achieve this goal six operated joints with residual symptoms radiographed one year post-operatively. Computed tomographic scanning with intravenous contrast, were examined. The direct volume rendering technique of CT images was used, and a data set of images to demonstrate the vasculature of the human Tempromandibular joint in three dimensions. After elaboration of the data through post-processing, the arterial supply of the Tempromandibular joint was compared to reference anatomy. The most commonly injured arteries are anterior tympanic, masseteric, superficial temporal arteries (detection rate 33\%, 33\%, 50\% respectively). Furthermore, osteoarthritic changes, synovial and articular cartilage calcifications with detached bony fragments were noticed that could be attributed to vascular injury. Open joint procedures are associated with significant decrease in arterial supply to TMJ, which could be correlated with osteoarthritic changes noticed later on. The three-dimensional volume rendering technique of computed tomography angiography is an effective non-invasive diagnostic tool; it allows simultaneous evaluation of boney changes and residual vascularity in previously operated joints.
\end{abstract}

Keywords: Revascularization, Temporomandibular Joint, Joint Surgery, CT Volume Rendering Technique

\section{Introduction}

The Tempromandibular joint (TMJ) is a ginglymoarthrodial synovial joint consisting of the mandibular condyle, squamous temporal bone, fibrous capsule with accessory ligaments and the synovial membrane. What makes this joint unique is the articular surfaces are covered by fibrocartilage instead of hyaline cartilage [1]

The blood supply to the TMJ is circumferential. Each vessel within a range of some three centimeters donates branches to the joint. The blood vessels to the TMJ mainly originate from the superficial temporal artery (STA, about $3.8 \mathrm{~mm}$ in diameter) and the maxillary artery (MA, about $3.2 \mathrm{~mm}$ in diameter) [2]. The MA is classified according to its relationship with the lateral pterygoid muscle and zygomatic arch. The superficial MA is located laterally to the lateral pterygoid muscle and with a distance between zygomatic arch and maxillary artery of $\geq 20 \mathrm{~mm}$. The deep MA is medial to the lateral pterygoid and with a distance between zygomatic arch and maxillary artery of $>20 \mathrm{~mm}[2,3]$.

Surgical interventions adopted in management of TMJ disorders range from non-invasive such as joint lavage to most invasive one. Clinicians usually prefer the most effective choice with minimal trauma in order to avoid disruption of nerve and blood supply.

Open joint surgery is usually associated with dissection of soft tissues and vascular injury, the severity of vascular injury could be linked with post-operative healing, remodeling as 
well as incidence of osteoarthosis and condylar resorption [4]. The present study performed to quantify the severity of vascular injury in an attempt to explain post-operative complications.

\section{Patients and Methods}

The present study reviewed the cervicocranial arterial supply of 6 operated joints whose presented with residual symptoms (pain \&/ or limitation of function) after one year of clinical follow-up. Four cases were included, all are females (two unilaterally operated and two bilaterally operated), age range from 23 to 45 years. They were examined using contrast-enhanced computed tomographic (CT) scanning in Ain shams university faculty of dentistry, department of oral and maxillofacial surgery, faculty of medicine department of diagnostic radiology, Cairo Egypt.

All of the subjects gave written informed consent at the beginning the study. The Medical Ethics Committee of the faculty of medicine approved the study protocol, which conformed to the principles of the Declaration of Helsinki for human subject research.

The examinations were performed with a 128-row CT scanner (PHILIPS Healthcare B. V, Ingenuity TP, Best, The Netherlands). The volume acquisition was performed during administration $100 \mathrm{~mL}$ of iodinated non-ionic contrast agent of $370 \mathrm{mg} / \mathrm{mL}$ concentration at right antecubital vein with an 18-gauge needle cannula using automatic injector (Medrad, Stellant TM, USA) with $5 \mathrm{~mL} / \mathrm{s}$ flow rate followed by $50 \mathrm{ml}$ saline in the same injection rate to optimize the arterial contrast opacification. To obtain best heightening of the arteries, the delay time between starting the contrast administration and scanning was assessed with the bolus test technique; venous district was intentionally overlooked, thus avoiding potential interference with the arterial images.

The acquisition volume used was extending in caudocranial direction from $\mathrm{C} 7$ to the sella turcica. The scan parameters were ) with the following scan parameters: $120 \mathrm{KV}, 250 \mathrm{mAs}$, beam collimation $160 * 0.5 \mathrm{~mm}$; rotation time $0.5 \mathrm{~s}$, pitch 0.89 ; with $1 \mathrm{~mm}$ slice thickness, image matrix $512 * 512$ and field of view, $229 \mathrm{~mm}$ with overall $4 \mathrm{sec}$ scan time.

For the rendering process, we used VolView 2.0 graphics software (Kitware Inc. Clifton Park, NY, USA). Specific algorithm wasz used by volume rendering system to transform conventional two-dimensional magnetic resonance imaging sets of slices transparent volume data set images and allowed visualization of data from numerous imaging modalities

The CT images were matched to the anatomic data acquired from cadaveric studies. The arterial branch on the CT images was categorized as traceable ( 2 points), recognizable only for a short trace ( 1 point), or unrecognizable ( 0 point). The total score for each artery was divided by 20 and multiplied by 100 to arrive at a depiction rate for each artery.

\section{Results}

Applying volume rendering techniques, after acquisition of source data, A volume 3D images of the temporomandibular region and related contrast enhanced vasculature were obtained then using surface shading and transparent techniques with bone subtraction to give more information and delineate the anatomical details and offer realistic views which permit evaluation of both bony and vascular structures. Then the operated TMJ related arteries and their branches are identified and compared to the normal anatomy as well as non-operated side (in unilaterally operated cases).

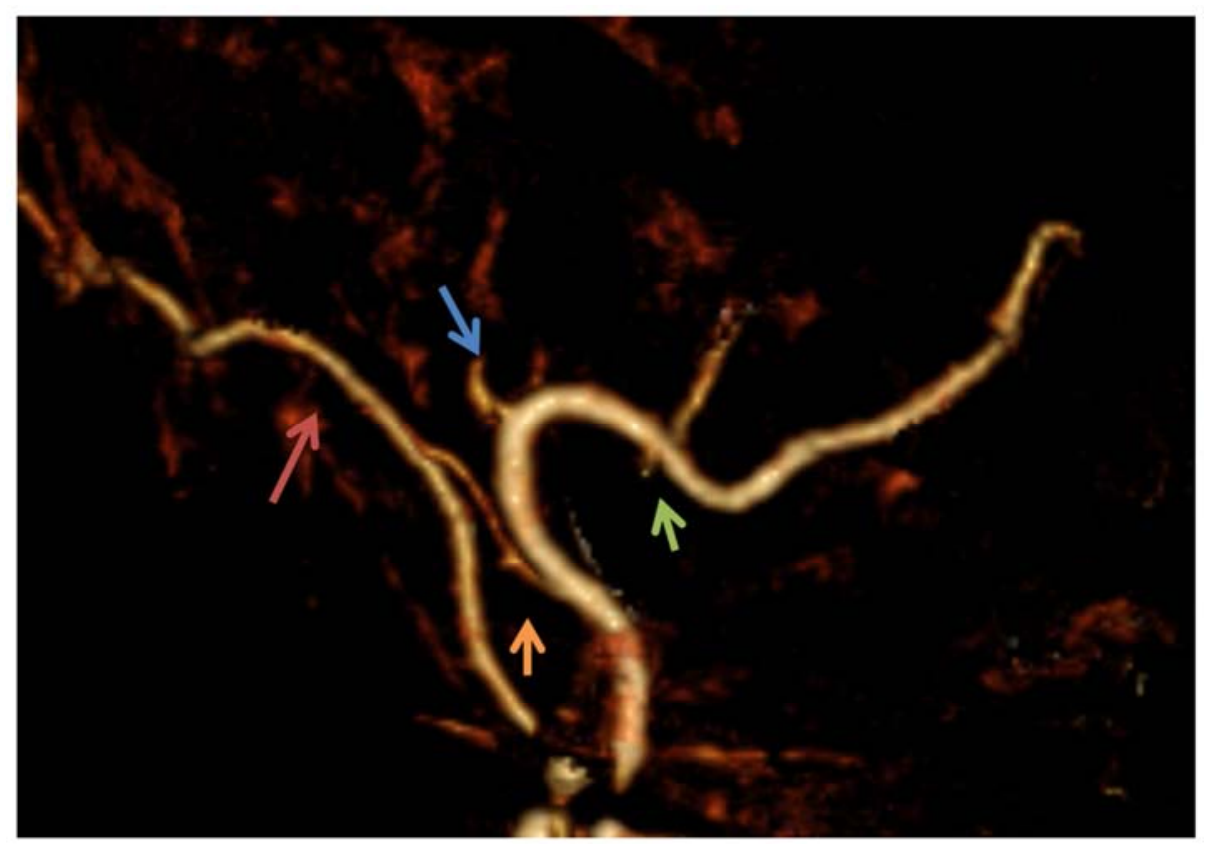

Figure 1. Posterior and lateral view after bone subtraction and rotating the image (Lateral angiograms), 3D image rotated more laterally for better visualization of external carotid branches medial to the condyle. Operated joint image of showing sharp ended both STA (blue arrow) and inferior dental arteries (green arrow) with intact Posterior auricular artery (orange arrow) Occipital artery (red arrow). 
Examining the temporomandibular region form poster lateral view, the ECA was seen easily cranially directed and after giving the posterior auricular artery, it curves laterally to run along the posterior ramus till space behind the neck of mandible, ending by dividing two major branches IMA and STA. Transverse facial artery (TFA), is seen arising from the STA passing anteriorly then disappeared into the parotid gland. Magnifications in the region of the mandibular condyle and adjustments in browsing parameters were done to observe the relations of the vessels to the bone structures especially the ECA, IMA and TFA (Figure 1).

After bone subtraction and rotating the image, more laterally, it was better to assess the mandibular branches of IMA (internal maxillary artery) around the TMJ, particularly, IDA (inferior dental artery), MMA (middle meningeal artery), AMA (accessory meningeal artery) and posterior auricular and occipital branches of ECA external carotid artery) (Figure 2).

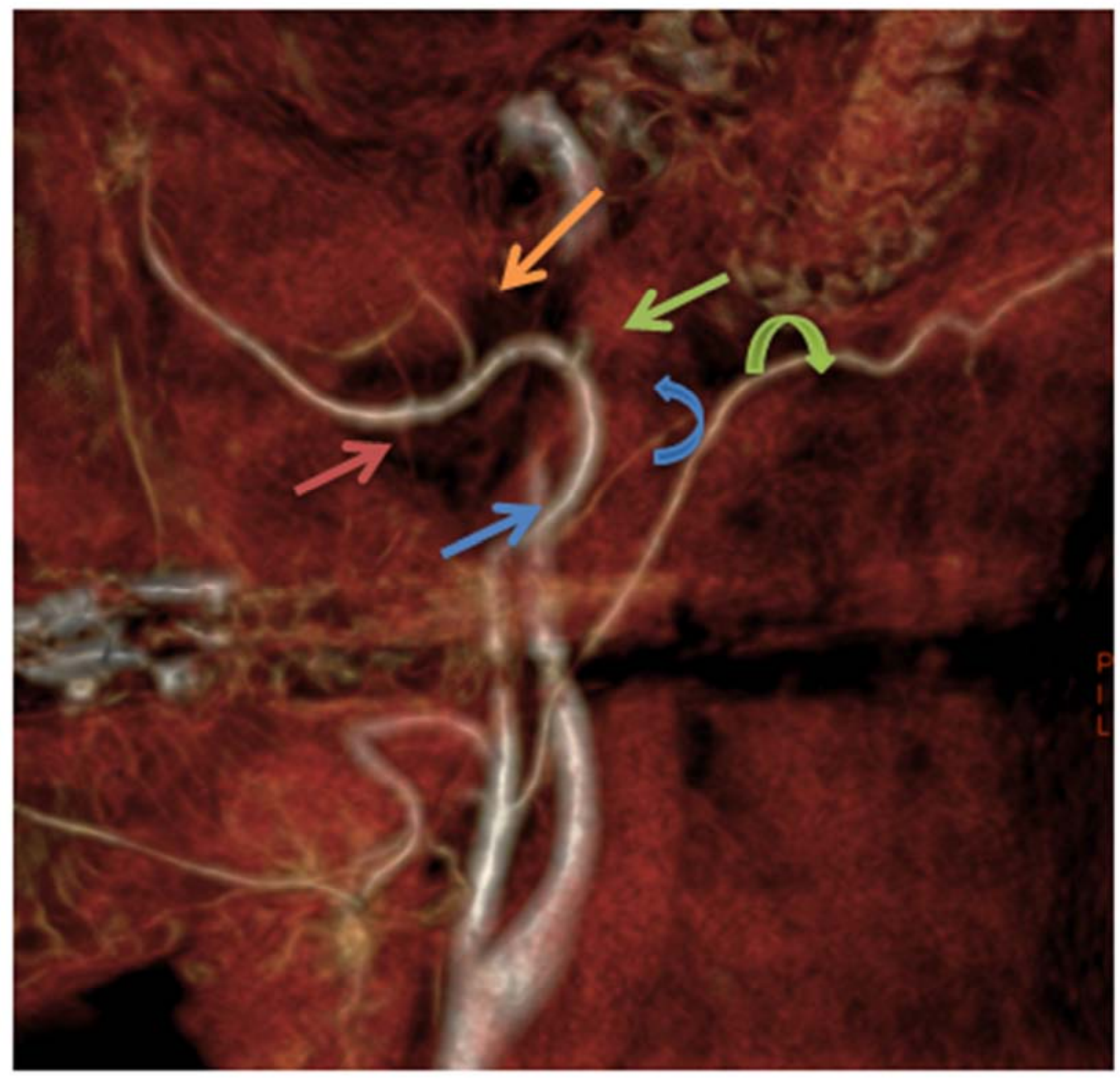

Figure 2. 3D volume rendering images of the left un-operated TMJ region. Maxillary A: Blue Arrow: ECA, green arrow: Superficial temporal A, orange arrow: common MMA and accessory meningeal artery trunk, red arrow: inferior alveolar A (inferior dental A). Blue and green curved arrows represent posterior auricular A and occipital Artery, branches of ECA. The patient has a superficial course of left internal maxillary artery so have common trunk of MMA and AMA (orange arrow).

In all patients in our study, The mandibular segment (first segment) of IMA starts vertically and then runs in horizontal pattern and then makes an acute turn anteriorly, that marks the transition to the second (zygomatic) segment and giving its branches which are ATA, MMA, AMA, and inferior dental (alveolar) artery IDA which were easily to be delineated in 3D image. Also, By using transparent techniques, it was able to delineate the IMA (internal maxillary artery), mandibular segment branches without bony subtraction. ATA is seen passing behind TMJ, reaching the external auditory canal. In our study, all detected ATA are seen arise from the mandibular branch of IMA and all TFA are from STA. Figure 3. 

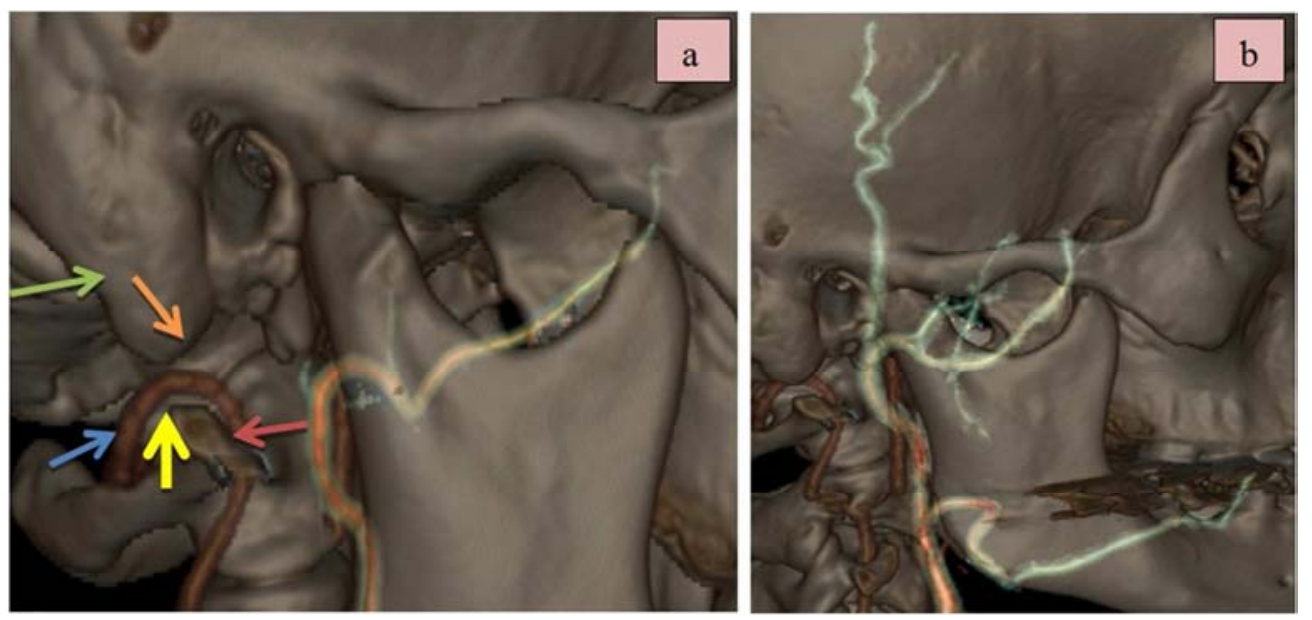

Figure 3. (a)Transparent Posterior-lateral view of computerized tomography rendering of TMJ a 33-year-old female (one year after disk replacement) showing severed superficial temporal, transverse facial, inferior dental arteries, intact internal maxillary a. (b): Transparent Posterior-lateral view of computerized tomography rendering of the temporomandibular joint un-operated right side with intact superficial temporal and internal maxillary arteries. The external carotid artery (blue a), the internal maxillary artery (yellow a), the superficial temporal artery (green a), the transverse facial artery (d), the inferior dental artery (red a), the middle meningeal artery (orang a)

Considering middle meningeal artery (MMA), it originates separately, or in common trunk with AMA, from the mandibular segment and directed cranially to enter foramen spinosum,. In our study, about $75 \%$ of the MMA and AMA arisen from a common trunk with superficial course of $2^{\text {nd }}$ segment of IMA and about $25 \%$ were of separate origin with deep course of the $2^{\text {nd }}$ segment. The inferior dental artery (IDA, f), is seen arising from the inferior aspect of mandibular segment and descends along medial mandibular ramus and entered the alveolar canal.

Examination of operated joints, after viewing the source images and tracing the vascular tree, was done, we found these postoperative features:

(a) All the detected ATA arise from the first part of maxillary artery. All the detected transverse facial artery arise from the STA

(b) Accidental finding: Two patients had osteoarthritic changes, One patient had surgical induced synovial and articular cartilage calcifications, bilateral, more at the left side, Few detached bony fragments are seen at most of the patients. One patient has right maxillary sinusitis with mycetema. Depiction rate of arteries in operated joints was as follow table 1

Table 1. Depiction rate of arterial blood supply in operated joints.

\begin{tabular}{ll}
\hline Artery & Depiction rate \% \\
\hline External Carotid Artery (ECA) & 100 \\
Superficial Temporal Artery (STA) & 50 \\
Internal Maxillary Artery (IMA) & 100 \\
Transverse Facial Artery (TFA) & 83 \\
Anterior Tympanic Artery (ATA) & 33 \\
Middle Meningeal Artery (MMA) & 100 \\
Accessory Meningeal Artery (AMA) & 67 \\
Inferior Dental Artery (IDA) & 83 \\
Masseteric Artery (MA) & 33 \\
Posterior Auricular (PAA) & 83 \\
Condylar branches of the Superficial Temporal Artery & 50 \\
\hline
\end{tabular}

\section{Discussion}

The vascular supply to TMJ is profuse. Each named vessel inside a radius of some three centimeters donates branches to the joint capsule. Major Arterial contributions come from the large superficial temporal and maxillary Arteries posteriorly, and the smaller posterior deep temporal, masseteric and Lateral pterygoid terminals anteriorly [5]. Open joint surgery through trans-cutaneous incisions is expected to be associated with disruption of unknown fraction of joint blood supply and possible nerve injury which has led to development of endoscopic surgery for certain applications to minimize complications. Sequel of diminished blood supply includes poor bone and soft tissue healing, TMJ arthrosis and idiopathic condylar resorption [6]

Although postsurgical revascularization of TMJ has been studied in rabbits [7] many years ago no studies have assessed postsurgical revascularization in human TMJ after open joint procedures. This study was performed to determine whether transcutaneous dissection and disruption of the joint capsule significantly affects postsurgical revascularization using volume rendering technique calculated from computerized tomograms with intra-venous contrast enhancement in symptomatic operated volunteers.

Cases investigated includes disc plication and discectomy which represent the most widely adopted treatment options in management of TMJ internal derangement disorders, especially those not responding to conservative protocols. Examination involves six operated joints all operated through endural incision by same operating team one year post-operatively to ensure adequate time for healing and re-vascularization after surgical trauma. Involved cases were those presented with residual symptoms (pain $+/$ - limitation) where CT examination helps in complete assessment of possible post-operative sequel.

Regarding selection of imaging method, Volume rendering 
technique has the principal of collection of series of axial images and construction of 3D images, which allows accurate depiction of 3D relationships, delineating the vascular anatomy and also defining the related soft tissue, bones and muscles. It gives more detailed information about the pathological conditions with advantage of color volume rendered images that facilitate understating the complex anatomy [8].

On the other hand MIP (Maximum projection) which could be used in vascular imaging, has less sensitivity and specificity where images affected by other high attenuation voxels (like calcified plaques or adjacent bone) that can obscure some vascular details. Also it gives no 3D detailed information about the related soft tissue or bony structure [9] In most of comparative studies in literature show that volume rendering technique is superior to MIP images in vascular imaging and considered an essential technique in $\mathrm{CT}$ angiography in all clinical applications $[10,11]$

Literature body showed a lot of studies that investigate vascularity of such anatomical region however no one has described post-surgical arterial injury [12-14]. Examination of unilaterally operated cases revealed the same arterial pattern previously described in literature and facilitates tracing of missed vessels. The most affected arteries are anterior tympanic ATA, masseteric MA, superficial temporal STA and condylar branch of superficial temporal arteries, which means significant reduction of superficial vascularity mostly due to trans-cutaneous approach and capsular incision.

Results of the present study suggest that vascular injury associated with endural incision, capsular incision, disc plication and discectomy is of significant severity, involving lateral and posterior vasculature. Osteoarthritic changes (seen in two cases), synovial and articular cartilage calcifications with detached bony fragments (seen in four cases) could be attributed to vascular injury together with surgical trauma. In fact post-surgical changes are multifactorial and not easy to correlate with certain etiology; however diminished blood supply is of significant importance in healing and remodeling scenario [6, 15] Modification in surgical approach and dissection technique could be suggested to minimize severity of vascular injury. Favorably, indications of open joint surgery are decreasing with invention of endoscope assisted procedure and interventional techniques. On the other hand the evident osteoarthritic changes seen in all cases could be attributed to diminished arterial blood supply however, future studies could investigate such critical finding.

\section{Conclusion}

Superficial vascularity (namely STA, ATA, MA and condylar branch of STA) is the most commonly injured vessels as a sequel of open joint procedure (discectomy and discoplasty) without post-operative revascularization. The three-dimensional volume rendering of computed tomography angiography is an effective non-invasive diagnostic tool especially in operated cases with persistent symptoms such as pain and limited mobility.

\section{References}

[1] McMinn, R. M. H.: Last. anatomy regional and applied In: Head and Neck and Spine. Churchill Livingstone, Edinburg, London, p. 523, (1994).

[2] Cuccia A, Caradonna C, Caradonna D, Anastasi G, Milardi D, Favaloro A, De Pietro A, Angileri T, Caradonna L, Cutroneo G. The arterial blood supply of the temporomandibular joint: an anatomical study and clinical Implications. Imaging Science in Dentistry $2013 ; 43$ : 37-44.

[3] Patnaik V. Sanju B; Rajan K. Anatomy of Temporomandibular Joint? A ReviewJ Anat. Soc. India 49(2) 191-197 (2000).

[4] Keith D. Complications of temporomandibular joint surgery. Oral Maxillofacial Surg Clin N Am 15 (2003) 187-194.

[5] Patnaik V. Sanju B; Rajan K. Anatomy Of Temporomandibular Joint? A ReviewJ Anat. Soc. India 49(2) 191-197 (2000).

[6] Hoffman, D, Puig L. Complications of TMJ. oral and maxillofacial surgery clinics September 2017.

[7] Cynthia R. Satko, T \& David I and Blaustein L. Postsurgical revascularization of the rabbit temporomandibular join A Histologic and Microangiographic Study. J Oral Maxillofac Surg 44:671-076.1966.

[8] Fishman EK, Ney DR. Heath DG, et al: $\quad$ Volume Rendering versus Maximum Intensity Projection in CT Angiography: What Works Best, When, and Why. Radio Graphics 2006; 26:905-922.

[9] The diagnostic contribution of CT volumetric rendering techniques in routine practice Simone Perandini, N Faccioli, A Zaccarella, TJ Re, and R Pozzi Mucelli Indian J Radiol Imaging. 2010 May; 20(2): 92-97.

[10] Hong KC, Freeny PC. Pancreaticoduodenal arcades and dorsal pancreatic artery: comparison of $\mathrm{CT}$ angiography with three-dimensional volume rendering, maximum intensity projection, and shaded surface display. AJR Am J Roentgenol 1999; 172:925-931.

[11] Addis KA, Hopper KD, Iyriboz TA, et al. CT angiography: in vitro comparison of five reconstruction methods. AJR Am J Roentgenol 2001; 177: 1171-1176.

[12] Uysal I; Buyukmumcu M; Dogan N; Seker M \& Ziylan T Clinical Significance of Maxillary Artery and its Branches: A Cadaver Study and Review of the Literature. Int. J. Morphol., 29(4):1274-1281, 2011.

[13] Takagi. R, westonson. P, ohashi. Y, togashi. H. Mr angiography of the tmj in asymptomatie volunteers. oral radiol. vol. 14 no. 2 1998(69 74).

[14] Kuruoglu E, Cokluk C, Marangoz A, Aydın K. The evaluation of three-dimensional anatomy of the superficial temporal artery using volume rendering technique. Turkish neurosurgery. march, 2014.

[15] Ingawale $\mathrm{S}$ and Goswami $\mathrm{T}$. temporomandibular joint: disorders, treatments, and biomechanics. Annals of Biomedical Engineering, Vol. 37, No. 5, May 2009 (_2009) pp. 976-996. 